\title{
USAGE OF MAGNETIC IRON TO RAISE TOLERANCE OF SOME ORNAMENTAL TREES AND SHRUBS TO SOIL SALINITY IN CASE OF HORSERADISH TREE (MORINGA OLEIFERA LAM.)
}

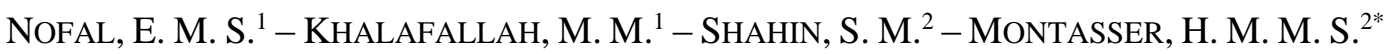 \\ ${ }^{1}$ Hort. Dept., Fac. Agric., Kafrelsheikh Univ., Egypt \\ ${ }^{2}$ Botanical Gards. Res. Dept., Hort. Res. Inst., ARC, Giza, Egypt \\ *Corresponding author \\ e-mail: heba.montaser78@gmail.com
}

(Received $20^{\text {th }}$ Sep 2020; accepted $19^{\text {th }}$ Jan 2021)

\begin{abstract}
An experiment was performed at Orman Botanical Garden, Giza, Egypt to determine the role of magnetite ore at either 2 or $4 \mathrm{~g} /$ pot in reducing the harmful effects of soil salinity on growth and quality of Moringa oleifera Lam. grown in soil mixture salinized with a mixture of $\mathrm{NaCl}$ and $\mathrm{CaCl}_{2}$ salts at concentrations of 0,2000, 4000, 6000 and $8000 \mathrm{ppm}$. The effect of the interaction between salinity and magnetite was also studied. The results indicated that a gradual decrease in survival percentage was observed with the gradual increments in salinity, but it was progressively increased with higher magnetite rates irrespective of salinity concentration. Connecting between magnetite at any dose and salinity up to $4000 \mathrm{ppm}$ gave the highest percentage of survival rate in the two seasons. While, the lowest survival rate was recorded at $8000 \mathrm{ppm}$, especially in the absence of magnetite. A response similar to that of survival percentage occurred for vegetative and root growth traits, and leaf chemical composition, with few exceptions in both seasons. Thus, it is advised to drench soil mixture with magnetite at $4 \mathrm{~g} / \mathrm{pot}$ to enhance growth and quality of Moringa oleifera transplants under salt stress.
\end{abstract}

Keywords: salinity, Moringa oleifera, magnetite, carotenoids, proline

\section{Introduction}

Among many species included in Fam. Moringaceae is considered the miracle species having tremendous uses, such as aforestation, alley cropping, medicines, water purification biopesticides, biogas, green manure and gum production, domestic cleaning agent and as a vegetable for their edible roots, young leaves, fruits and seeds, from which oil is often extracted (Aiyelaagbe, 2011). It grows rapidly up to 9-10 m height in poor soil with little care for long periods of drought (McConnachie, 1999). It is originated in India and Pakistan, but is now distributed to most tropical areas as ornamental, fodder, fibrous, malnutrition solving and economic plant (Fahey, 2005; Thurber and Fahey, 2009). It is easily propagated by seeds and stem cuttings, tolerates a wide range of soil conditions, but prefers a neutral to slightly acidic ( $\mathrm{pH}$ 6.5-7), well drained sandy or loamy soils. It is considered as drought resistant, but water logging sensitive (Suein, 2008).

Regarding salinity tolerance of Moringa oleifera, Dos Santos et al. (2011) noticed that the gradual increase in $\mathrm{NaCl}$ concentration $\left(0,25,50,100,200\right.$ and $\left.250 \mathrm{~mol} / \mathrm{m}^{3}\right)$ caused a gradual decrement in germination \% germination speed index and seedling length. Moreover, Nouman et al. (2012a) reported that moringa seedlings survived up to $3500 \mathrm{ppm} \mathrm{NaCl}$ salinity with a slight reduction in its biomass, chlorophyll a, crude protein and mineral concentrations. Activity of superoxide dismutase (SOD) and peroxidase (POD) and $\mathrm{Ca}, \mathrm{K}$ and $\mathrm{Mg}$ concentrations were progressively decreased, but 
catalase (CAT), total phenols, chlorophyll b, $\beta$-carotene, $\mathrm{Na}$ and $\mathrm{P}$ concentrations were significantly increased with raising salinity up to $5500 \mathrm{ppm}$ relative to control. Similarly, were those results of Nouman et al. (2012b), Rivas et al. (2013) and Elhag and Abdalla (2014) on Moringa oleifera.

The deleterious effects of salinity on ornamentals, such as reduction of plant growth and leaf yellowing, browning of the leaf tips and margins, defoliation, root shortening and may ultimately plant die were previously suggested by Al-Qubaie et al. (2003) on Bongainvilleaglabra, Conocarpus erectus, Ficus benghalensis, Jasminum azoricum, Tamarix articulata and Ziziphus spina-christi, Shahin et al. (2008) on Ficus macrocarpavar. Hawaii and Euonymus Joponica cv. Mediopicta, Shahin et al. (2013) on Ficus benjamina cv. Samantha and Schefflera arboricola cv. Gold Capella, El-Fouly et al. (2015) on Iris tingitana cv. Wedgewood, Jose et al. (2016) on Eucalyptus urophylla and Shahin et al. (2017) on Casuarina equisetifolia and Eucalyptus rostrata. However, these harms were minimized by some practical methods, such as those revealed by Moustafa et al. (2017) on Moringa oleifera using magnetic iron. The role of magnetite in reducing hazards of salinity was also documented by Abdel-Fattah (2014) on Jacaranda acutifolia, Ahmed et al. (2016) on Acalypha wilkesiana, Shahin et al. (2018) on Terminalia arjuna and El-Sayed et al. (2019) on Enterolobium contortisiliquum.

Such trial, however aims to evaluate the role of magnetic iron in reducing the harmful effects of soil salinity on growth and quality of Moringa oleifera transplants during rearing period in the nursery.

\section{Materials and methods}

The current study was conducted in the full sun the average temperature between $\left(27-38^{\circ} \mathrm{C}\right)$ and the average percentage of humidity $56 \%$ at the nursery of Orman Botanical garden, Giza, Egypt throughout the two successive seasons of 2018 and 2019 as an attempt to verify the benefit of magnetite in minimizing the hazards of soil salinity on growth and quality of moringa transplants, and to detect its role in helping these nurslings to grow well under such stress.

Thus, 4-months-old, uniform transplants of the plant at a length of about $30 \mathrm{~cm}$ with 3-4 leaves were transplanted on April, $1^{\text {st }}$ for every season to 15 -cm-diameter polyethylene black bags (one seedling/bag) filled with about $3.5 \mathrm{~kg}$ of sand and clay mixture at equal volume parts $(1: 1, \mathrm{v} / \mathrm{v})$. The physical and chemical properties of the soil mixture used in the two seasons were determined and illustrated in Table 1.

Table 1. The physical and chemical analysis of the soil mixture used in 2018 and 2019 seasons

\begin{tabular}{|c|c|c|c|c|c|c|c|c|c|c|c|}
\hline Soil mixture & \multicolumn{4}{|c|}{ Particle size distribution (\%): } & \multirow{2}{*}{ S.P. } & \multirow{2}{*}{$\begin{array}{l}\text { E.C. } \\
(\mathrm{dS} / \mathrm{m})\end{array}$} & \multirow{2}{*}{ pH } & \multicolumn{4}{|c|}{ Cations (meq/l) } \\
\hline \multirow{2}{*}{$\begin{array}{c}\text { Sand + Clay } \\
(1: 1, \mathrm{v} / \mathrm{v})\end{array}$} & Coarse sand & Fine sand & Silt & Clay & & & & $\mathrm{Ca}^{++}$ & $\mathrm{Mg}^{++}$ & $\mathrm{Na}^{+}$ & $\mathrm{K}^{+}$ \\
\hline & 38.6 & 31.3 & 20.6 & 9.5 & 25 & 6.5 & 7.86 & 17.8 & 14.2 & 33.1 & 0.9 \\
\hline \multirow{2}{*}{ Soil texture } & \multicolumn{4}{|c|}{ Anions (meq/l) } & \multicolumn{7}{|c|}{ Macro-and micro-elements (ppm) } \\
\hline & $\mathrm{HCO}_{3}^{-}$ & $\mathrm{Cl}^{-}$ & $\mathrm{SO}_{4}^{--}$ & $\mathrm{CO}_{3}$ & $\mathrm{~N}$ & $\mathrm{P}$ & $\mathrm{K}$ & $\mathrm{Fe}$ & $\mathrm{Zn}$ & $\mathrm{Mn}$ & $\mathrm{Cu}$ \\
\hline Sandy clay & 2.10 & 58.40 & 5.50 & 0.00 & 173.10 & 15.78 & 361.76 & 15.80 & 4.36 & 8.03 & 8.81 \\
\hline
\end{tabular}


Immediately before transplanting, the above soil mixture was salinized with an equal mixture of $\mathrm{NaCl}$ and $\mathrm{CaCl}_{2}$ pure salts $(1: 1$, by weight) at the concentrations of 0,2000 , 4000, 6000 and $8000 \mathrm{ppm}$. After planting, however the soil mixture was thoroughly drenched with magnetic iron $\left(\mathrm{Fe}_{3} \mathrm{O}_{4}, 22.5 \%\right)$ secured from Alahram Mining Co., Maadi, Cairo at the rates of 0,2 and $4 \mathrm{~g} / \mathrm{plant}$, as one batch at the beginning of the season. Each level of salinity was combined with each one of magnetic iron to formalize 15 interaction treatments.

The transplants under various experimental treatments were fertilized 3 times during the course of this study with $2 \mathrm{~g} /$ transplants of NPK + micro elements chemical fertilizer (Kristalon, 19:19:19) and watered every other day. Furthermore, all the other agricultural practices needed for such plantation were done as usually grower did. A factorial in complete randomized design with 3 replicates, as each one contained 5 seedlings was used in every season (Mead et al., 1993).

Data recorded, at the end of each season (on October, $1^{\text {st }}$ ), were: survival (\%), plant height $(\mathrm{cm})$, stem diameter $(\mathrm{cm})$, number of leaves/plant, leaf length $(\mathrm{cm})$, the longest root length $(\mathrm{cm})$, root collar diameter $(\mathrm{cm})$, aerial parts and roots fresh and dry weights $(\mathrm{g})$ and salt resistance index as a percentage (SRI \%) was calculated from the equation of Wu and Huff (1983) as follows:

SRI $(\%)=$ Mean root length of the longest root in salt treated plant / mean root length of the longest root in control one $\mathrm{x} 100$

In addition, the benefit coefficient of magnetite $\left(\mathrm{Fe}_{3} \mathrm{O}_{4}\right)$ under various salinity levels as a percentage (B. Coe \%) was estimated from the following equation:

B. Coe $(\%)=$ Increase rate in dry matter of treated plant / dry matter of control one $\mathrm{x} 100$.

In fresh samples concentrations of photosynthetic pigments (chlorophyll $a, b$ and carotenoids, mg/g f.w.) were determined according to the method of Sumanta et al. (2014), while in dry ones the percentages of nitrogen (Blake, 1965), phosphorus (Luatanab and Olsen, 1965), as well as potassium, sodium and chloride (Jackson, 1973) were measured. Concentration of the free proline $(\mathrm{mg} / 100 \mathrm{~g} \mathrm{~d} . \mathrm{w}$.) was also assessed in dry leaf samples by the method of Batels et al. (1973). The abovementioned constituents were evaluated in the second season only.

Data were then subjected to analysis of variance using the computer program of SAS Institute (2009), followed by Duncan's New Multiple Range t-Test (Steel and Torrie, 1980) for means comparison.

\section{Results}

\section{Effect of soil salinity, magnetite and their interaction on vegetative and root growth parameters}

Data presented in Table 2 show that survival \% means were descendingly decreased with increasing salinity level to reach the minimum at $8000 \mathrm{ppm}$ level in both seasons, but progressively increased with rising magnetite rate to be maximum at $4 \mathrm{~g} / \mathrm{plant}$ rate 
regardless of salinity level. Combining between any dose of magnetic iron and salinity up to $4000 \mathrm{ppm}$ gave the highest survival percentage in the two seasons. A significant reduction in means of this character was observed when salinity level was elevated to 6000 ppm, but it was still higher than 50\% in most cases of both seasons especially in the presence of magnetite. The least records however were attained by $8000 \mathrm{ppm}$ salinity level, even in the presence of $\mathrm{Fe}_{3} \mathrm{O}_{4}$, but it's absence greatly reduced means of such trait to $27.4 \%$ in the first season and to $00.00 \%$ in the second one due to death of the plants.

Table 2. Effect of salinity levels, magnetite rates and their interactions on survival and height of Moringa oleifera Lam. transplants during 2018 and 2019 seasons

\begin{tabular}{|c|c|c|c|c|c|c|c|c|}
\hline \multirow{3}{*}{ Salinity level (ppm) } & \multicolumn{4}{|c|}{ Survival (\%) } & \multicolumn{4}{|c|}{ Plant height (cm) } \\
\hline & 0.00 & $2 \mathrm{~g}$ & $4 \mathrm{~g}$ & Mean & 0.00 & $2 \mathrm{~g}$ & $4 \mathrm{~g}$ & Mean \\
\hline & \multicolumn{8}{|c|}{ First season; 2018} \\
\hline 0.00 & $100.00 \mathrm{a}$ & $100.00 \mathrm{a}$ & $100.00 \mathrm{a}$ & $100.00 \mathrm{~A}$ & $94.67 d$ & $103.0 \mathrm{~b}$ & $108.9 \mathrm{a}$ & $102.2 \mathrm{~A}$ \\
\hline 2000 & $100.00 \mathrm{a}$ & $100.00 \mathrm{a}$ & $100.00 \mathrm{a}$ & $100.00 \mathrm{~A}$ & $92.00 \mathrm{e}$ & $98.99 \mathrm{c}$ & $102.6 \mathrm{~b}$ & $97.86 \mathrm{~B}$ \\
\hline 4000 & $89.22 b$ & $100.00 \mathrm{a}$ & $100.00 \mathrm{a}$ & $96.41 \mathrm{~B}$ & $70.78 \mathrm{i}$ & $78.93 \mathrm{~h}$ & $88.51 \mathrm{f}$ & $79.41 \mathrm{C}$ \\
\hline 6000 & $53.17 \mathrm{e}$ & $70.82 d$ & $76.91 \mathrm{c}$ & $66.97 \mathrm{C}$ & $62.12 \mathrm{j}$ & $70.17 \mathrm{i}$ & $83.11 \mathrm{~g}$ & $71.80 \mathrm{D}$ \\
\hline 8000 & $27.40 \mathrm{~g}$ & $35.17 f$ & $40.34 \mathrm{f}$ & $34.30 \mathrm{D}$ & 49.001 & $55.73 \mathrm{k}$ & $63.10 \mathrm{j}$ & $55.95 \mathrm{E}$ \\
\hline \multirow[t]{2}{*}{ Mean } & $73.96 \mathrm{C}$ & $81.20 \mathrm{~B}$ & $83.45 \mathrm{~A}$ & & $73.71 \mathrm{C}$ & $81.36 \mathrm{~B}$ & $89.25 \mathrm{~A}$ & \\
\hline & \multicolumn{8}{|c|}{ Second season; 2019} \\
\hline 0.00 & $100.00 \mathrm{a}$ & $100.00 \mathrm{a}$ & $100.00 \mathrm{a}$ & $100.00 \mathrm{~A}$ & $86.50 \mathrm{e}$ & $103.0 \mathrm{~b}$ & $114.4 \mathrm{a}$ & $101.8 \mathrm{~A}$ \\
\hline 2000 & $100.00 \mathrm{a}$ & $100.00 \mathrm{a}$ & $100.00 \mathrm{a}$ & $100.00 \mathrm{~A}$ & $84.83 \mathrm{ef}$ & $100.1 \mathrm{c}$ & $102.2 b c$ & $95.71 \mathrm{~B}$ \\
\hline 4000 & $73.33 c$ & $87.20 b$ & $100.00 \mathrm{a}$ & 86.94B & $76.67 \mathrm{~g}$ & $83.67 f$ & $94.43 d$ & $84.92 \mathrm{C}$ \\
\hline 6000 & $41.53 \mathrm{f}$ & $58.30 \mathrm{e}$ & $65.80 \mathrm{~d}$ & $55.21 \mathrm{C}$ & $64.50 \mathrm{i}$ & $73.90 \mathrm{~h}$ & $87.27 \mathrm{e}$ & $75.22 \mathrm{D}$ \\
\hline 8000 & $0.00 \mathrm{~h}$ & $34.50 \mathrm{~g}$ & $38.30 \mathrm{~g}$ & 24.27D & 0.001 & $33.07 \mathrm{k}$ & $36.10 \mathrm{j}$ & $23.06 \mathrm{E}$ \\
\hline Mean & $62.97 \mathrm{C}$ & $76.00 \mathrm{~B}$ & $80.82 \mathrm{~A}$ & & $62.50 \mathrm{C}$ & $79.01 \mathrm{~B}$ & $86.89 \mathrm{~A}$ & \\
\hline
\end{tabular}

Mean followed by the same letter in a column or row don't differ significantly according to Duncan's New Multiple Range test

A similar trend to that of survival percentage was just obtained regarding plant height $(\mathrm{cm})$, stem diameter $(\mathrm{cm})$, No. leaves/plant, leaf length $(\mathrm{cm})$, root length $(\mathrm{cm})$, root collar diameter $(\mathrm{cm})$, as well as aerial parts and roots fresh and dry weights $(\mathrm{g})$ attributes (Tables 2, 3, 4, 5 and 6), as the means of these traits were gradually decreased with increasing salinity levels, but progressively increased with raising magnetic iron rate. The highest averages were also recorded by the concentrations between salinity concentration up to $4000 \mathrm{ppm}$ and any dose of magnetite. Increasing salinity level to $6000 \mathrm{ppm}$ caused a significant reduction in the means of foregoing criteria, with the exception for plants which received $\mathrm{Fe}_{3} \mathrm{O}_{4}$ at any rate. The minimal values were however acquired by $8000 \mathrm{ppm}$ salinity treatments especially for plants abandoned of magnetic iron.

\section{Salt resistance index (SRI \%) and benefit coefficient of magnetite (B. Coe. \%).}

It is clear from data presented in Table 7 that percent of salt resistance index was linearly decreased as salinity concentration was increased. Thus, the least SRI value was achieved by $8000 \mathrm{ppm}$ concentration to be $53.54 \%$ in the first season and $39.46 \%$ in the 
second one. Decrement mean of this index to less than $50 \%$ really indicates the low tolerance of moringa to salinity higher than $6000 \mathrm{ppm}$. Magnetite at the rate of $2 \mathrm{~g} / \mathrm{plant}$ scored the highest percentages of SRI in the two seasons, followed by $4 \mathrm{~g} / \mathrm{plant}$ rate. Under control treatment, a pronounced decline in the values of this index was acquired with increasing salinity level to be less than $50 \%$ in both seasons by 8000 ppm treatment. This confirms that moringa is not able to tolerate salinity higher than $6000 \mathrm{ppm}$ as mentioned before. On the other side, application of $\mathrm{Fe}_{3} \mathrm{O}_{4}$ improved the means of such index to be higher than $50 \%$ even under 8000 ppm treatment, showing the important role of this natural ore in enhancing moringa tolerance to high salinity.

Table 3. Effect of salinity levels, magnetite rates and their interactions on stem diameter and No. leaves/plant of Moringa oleifera Lam. transplants during 2018 and 2019 seasons

\begin{tabular}{|c|c|c|c|c|c|c|c|c|}
\hline \multirow{3}{*}{ Salinity level (ppm) } & \multicolumn{4}{|c|}{ Stem diameter $(\mathrm{cm})$} & \multicolumn{4}{|c|}{ No. leaves /plant } \\
\hline & 0.00 & $2 \mathrm{~g}$ & $4 \mathrm{~g}$ & Mean & 0.00 & $2 \mathrm{~g}$ & $4 \mathrm{~g}$ & Mean \\
\hline & \multicolumn{8}{|c|}{ First season; 2018} \\
\hline 0.00 & $0.797 \mathrm{c}$ & $0.870 \mathrm{~b}$ & $0.947 \mathrm{a}$ & $0.871 \mathrm{~A}$ & $7.07 \mathrm{c}$ & $8.33 b$ & $9.67 \mathrm{a}$ & $8.36 \mathrm{~A}$ \\
\hline 2000 & $0.740 \mathrm{~d}$ & $0.830 \mathrm{c}$ & $0.890 \mathrm{~b}$ & 0.820B & $6.50 \mathrm{~d}$ & $8.50 \mathrm{~b}$ & $9.50 \mathrm{a}$ & $8.17 \mathrm{~B}$ \\
\hline 4000 & $0.683 \mathrm{ef}$ & $0.737 d$ & $0.803 c$ & $0.741 \mathrm{C}$ & $5.67 \mathrm{e}$ & $7.17 \mathrm{c}$ & $8.33 b$ & $7.06 \mathrm{C}$ \\
\hline 6000 & $0.577 \mathrm{~g}$ & $0.647 f$ & $0.693 \mathrm{e}$ & $0.639 \mathrm{D}$ & $3.83 \mathrm{~g}$ & $5.67 \mathrm{e}$ & $6.50 \mathrm{~d}$ & 5.33D \\
\hline 8000 & $0.477 \mathrm{i}$ & $0.497 \mathrm{i}$ & $0.527 \mathrm{~h}$ & $0.500 \mathrm{E}$ & $3.00 \mathrm{~h}$ & $4.00 \mathrm{~g}$ & $4.83 f$ & $3.94 \mathrm{E}$ \\
\hline \multirow[t]{2}{*}{ Mean } & $0.655 \mathrm{C}$ & $0.716 \mathrm{~B}$ & $0.772 \mathrm{~A}$ & & $5.21 \mathrm{C}$ & $6.33 \mathrm{~B}$ & 7.77A & \\
\hline & \multicolumn{8}{|c|}{ Second season; 2019} \\
\hline 0.00 & $0.750 \mathrm{de}$ & $0.877 b$ & 0.930a & $0.852 \mathrm{~A}$ & $7.33 \mathrm{e}$ & $8.67 \mathrm{c}$ & $9.67 \mathrm{a}$ & $8.56 \mathrm{~A}$ \\
\hline 2000 & $0.740 \mathrm{de}$ & $0.837 b c$ & $0.863 b$ & $0.813 \mathrm{~A}$ & $5.33 \mathrm{~g}$ & $9.00 \mathrm{c}$ & $9.33 \mathrm{ab}$ & $7.89 \mathrm{~B}$ \\
\hline 4000 & $0.660 f$ & $0.713 \mathrm{e}$ & $0.793 \mathrm{~cd}$ & $0.722 \mathrm{~B}$ & $5.67 \mathrm{~g}$ & $7.00 \mathrm{ef}$ & $8.00 \mathrm{~d}$ & $6.89 \mathrm{C}$ \\
\hline 6000 & $0.547 \mathrm{~g}$ & $0.627 f$ & $0.730 \mathrm{e}$ & $0.634 \mathrm{C}$ & $4.67 \mathrm{~h}$ & $5.67 \mathrm{~g}$ & $6.67 f$ & $5.67 \mathrm{D}$ \\
\hline 8000 & $0.00 \mathrm{i}$ & $0.353 \mathrm{~h}$ & $0.387 \mathrm{~h}$ & $0.247 \mathrm{D}$ & $0.00 \mathrm{j}$ & $3.33 \mathrm{i}$ & $4.67 \mathrm{~h}$ & $2.67 \mathrm{E}$ \\
\hline Mean & $0.539 \mathrm{C}$ & $0.681 \mathrm{~B}$ & $0.741 \mathrm{~A}$ & & $4.60 \mathrm{C}$ & $6.73 \mathrm{~B}$ & $7.67 \mathrm{~A}$ & \\
\hline
\end{tabular}

Mean followed by the same letter in a column or row don't differ significantly according to Duncan's New Multiple Range test

As for the benefit coefficient of magnetite percentage (B. Coe. \%), as a real indicator for the advantage of magnetic iron in reducing hazards of salinity, with promoting plant growth, is presented in Table 7 which exhibits that $\mathrm{Fe}_{3} \mathrm{O}_{4}$ significantly increased dry matter production of treated plants under the different salinity levels over control in the two seasons. However, the upper hand in the increased means of such coefficient in the first season was for magnetite under 2000 and $6000 \mathrm{ppm}$ salinity treatments, as 31.88 and $31.27 \%$ against $15.97 \%$ for control, followed by magnetite under 4000 ppm level, while in the second season, that was true for magnetite under $8000 \mathrm{ppm}$ level, which raised the percent of this coefficient to $259.0 \%$ versus $27.85 \%$ for control. Likewise, a progressive increment was occurred in the means of this coefficient with raising $\mathrm{Fe}_{3} \mathrm{O}_{4}$ rate. So, the greatest benefit of this ore was recorded in both seasons by $4 \mathrm{~g} / \mathrm{plant}$ dose. Interaction treatments also encouraged dry matter production as applying $\mathrm{Fe}_{3} \mathrm{O}_{4}$ at 2 or $4 \mathrm{~g} /$ plant caused a significant increase in the production of dry matter over control regardless of salinity level in the two seasons. 
Table 4. Effect of salinity levels, magnetite rates and their interactions on leaf and root length and root callar diameter of Moringa oleifera Lam. transplants during 2018 and 2019 seasons

\begin{tabular}{|c|c|c|c|c|c|c|c|c|c|c|c|c|}
\hline \multirow{3}{*}{ Salinity level (ppm) } & \multicolumn{4}{|c|}{ Leaf length $(\mathrm{cm})$} & \multicolumn{4}{|c|}{ Root length $(\mathrm{cm})$} & \multicolumn{4}{|c|}{ Root callar diameter (cm) } \\
\hline & 0.00 & $2 \mathrm{~g}$ & $4 \mathrm{~g}$ & Mean & 0.00 & $2 \mathrm{~g}$ & $4 \mathrm{~g}$ & Mean & 0.00 & $2 \mathrm{~g}$ & $4 \mathrm{~g}$ & Mean \\
\hline & \multicolumn{12}{|c|}{ First season; 2018} \\
\hline 0.00 & $15.81 \mathrm{~g}$ & $14.94 h$ & $16.11 \mathrm{fg}$ & $15.62 \mathrm{C}$ & $13.44 \mathrm{~cd}$ & $13.97 \mathrm{bc}$ & $16.19 \mathrm{a}$ & $14.53 \mathrm{~A}$ & $1.87 \mathrm{de}$ & $2.20 \mathrm{c}$ & $2.37 \mathrm{~b}$ & $2.14 \mathrm{~B}$ \\
\hline 2000 & $16.67 \mathrm{ef}$ & $18.78 \mathrm{~d}$ & $20.50 \mathrm{~b}$ & $18.65 \mathrm{~A}$ & $12.49 \mathrm{e}$ & $13.07 \mathrm{~d}$ & $14.37 \mathrm{~b}$ & 13.31B & $1.80 \mathrm{ef}$ & $2.40 \mathrm{~b}$ & $2.47 \mathrm{~b}$ & $2.22 \mathrm{AB}$ \\
\hline 4000 & $11.90 \mathrm{i}$ & $19.45 c$ & $21.72 \mathrm{a}$ & 17.69B & $10.92 \mathrm{f}$ & $11.44 \mathrm{f}$ & $13.35 \mathrm{~d}$ & $11.91 \mathrm{C}$ & $1.67 \mathrm{fg}$ & $2.27 \mathrm{~b}$ & $2.67 \mathrm{a}$ & $2.27 \mathrm{~A}$ \\
\hline 6000 & $10.78 \mathrm{j}$ & $16.06 \mathrm{~g}$ & $17.23 \mathrm{e}$ & 14.69D & $8.86 h$ & $9.93 \mathrm{~g}$ & $10.93 f$ & $9.91 \mathrm{D}$ & $1.50 \mathrm{~g}$ & $1.92 \mathrm{de}$ & $1.99 \mathrm{~d}$ & $1.80 \mathrm{C}$ \\
\hline 8000 & 9.031 & $9.65 \mathrm{k}$ & $10.80 \mathrm{j}$ & $9.83 \mathrm{E}$ & $6.10 \mathrm{j}$ & $8.20 \mathrm{i}$ & $9.05 \mathrm{~h}$ & $7.78 \mathrm{E}$ & $1.27 \mathrm{~h}$ & $1.56 \mathrm{~g}$ & $1.61 \mathrm{~g}$ & $1.48 \mathrm{D}$ \\
\hline \multirow[t]{2}{*}{ Mean } & $12.84 \mathrm{C}$ & $15.78 \mathrm{~B}$ & $17.27 \mathrm{~A}$ & & $10.36 \mathrm{C}$ & $11.32 \mathrm{~B}$ & $12.78 \mathrm{~A}$ & & $1.62 \mathrm{C}$ & $2.11 \mathrm{~B}$ & $2.22 \mathrm{~A}$ & \\
\hline & \multicolumn{12}{|c|}{ Second season; 2019} \\
\hline 0.00 & $15.55 b$ & $16.96 a$ & $17.25 \mathrm{a}$ & $16.59 \mathrm{~A}$ & $12.50 \mathrm{c}$ & $13.22 \mathrm{~b}$ & $15.36 \mathrm{a}$ & $13.70 \mathrm{~A}$ & $2.20 \mathrm{bc}$ & $2.24 b$ & $2.57 \mathrm{a}$ & $2.34 \mathrm{~A}$ \\
\hline 2000 & $14.19 \mathrm{c}$ & $15.15 b$ & $17.28 \mathrm{a}$ & 15.54B & $11.44 d$ & $11.52 \mathrm{~d}$ & $13.45 \mathrm{~b}$ & 12.14B & $2.02 \mathrm{~d}$ & $2.04 \mathrm{~cd}$ & $2.37 \mathrm{~b}$ & $2.14 \mathrm{~B}$ \\
\hline 4000 & $13.06 \mathrm{~d}$ & $14.17 \mathrm{c}$ & $15.25 b$ & $14.16 \mathrm{C}$ & $10.16 \mathrm{f}$ & $10.78 \mathrm{e}$ & $13.00 \mathrm{c}$ & $11.31 \mathrm{C}$ & $1.79 \mathrm{e}$ & $1.90 \mathrm{de}$ & $2.31 b$ & $2.00 \mathrm{C}$ \\
\hline 6000 & $11.94 \mathrm{e}$ & $11.42 \mathrm{ef}$ & $14.00 \mathrm{c}$ & $12.45 \mathrm{D}$ & $8.31 \mathrm{~h}$ & $8.94 \mathrm{~g}$ & $10.03 \mathrm{f}$ & $9.09 \mathrm{D}$ & $1.45 \mathrm{~g}$ & $1.58 \mathrm{fg}$ & $1.74 \mathrm{ef}$ & $1.59 \mathrm{D}$ \\
\hline 8000 & $0.00 \mathrm{~h}$ & $8.06 \mathrm{~g}$ & $11.33 \mathrm{f}$ & $6.47 \mathrm{E}$ & $0.00 \mathrm{i}$ & $8.11 \mathrm{~h}$ & $8.61 \mathrm{gh}$ & $5.57 \mathrm{E}$ & $0.00 \mathrm{~h}$ & $1.43 \mathrm{~g}$ & $1.48 \mathrm{~g}$ & $0.97 \mathrm{E}$ \\
\hline Mean & $10.95 \mathrm{C}$ & $13.15 \mathrm{~B}$ & $15.02 \mathrm{~A}$ & & $8.48 \mathrm{C}$ & $10.51 \mathrm{~B}$ & $12.09 \mathrm{~A}$ & & $1.49 \mathrm{C}$ & $1.84 \mathrm{~B}$ & $2.09 \mathrm{~A}$ & \\
\hline
\end{tabular}

Mean followed by the same letter in a column or row don't differ significantly according to Duncan's New Multiple Range test 


$$
\text { - } 1183 \text { - }
$$

Table 5. Effect of salinity levels, magnetite rates and their interactions on aerial parts fresh and dry weights of Moringa oleifera Lam. transplants during 2018 and 2019 seasons

\begin{tabular}{|c|c|c|c|c|c|c|c|c|}
\hline \multirow{3}{*}{ Salinity level (ppm) } & \multicolumn{4}{|c|}{ Aerial parts fresh weights (g) } & \multicolumn{4}{|c|}{ Aerial parts dry weights (g) } \\
\hline & 0.00 & $2 \mathrm{~g}$ & $4 \mathrm{~g}$ & Mean & 0.00 & $2 \mathrm{~g}$ & $4 \mathrm{~g}$ & Mean \\
\hline & \multicolumn{8}{|c|}{ First season; 2018} \\
\hline 0.00 & $13.64 \mathrm{de}$ & $14.17 d$ & $19.44 \mathrm{a}$ & $15.75 \mathrm{~A}$ & $6.37 \mathrm{c}-\mathrm{e}$ & $6.94 c$ & $8.48 \mathrm{a}$ & $7.27 \mathrm{~A}$ \\
\hline 2000 & $10.95 \mathrm{f}$ & $15.19 \mathrm{c}$ & $17.60 \mathrm{~b}$ & $14.58 \mathrm{~B}$ & $5.15 \mathrm{f}$ & $6.98 \mathrm{c}$ & $7.63 b$ & $6.59 \mathrm{~B}$ \\
\hline 4000 & $9.80 \mathrm{~g}$ & $13.12 \mathrm{e}$ & $15.44 \mathrm{c}$ & $12.78 \mathrm{C}$ & $4.59 \mathrm{fg}$ & $6.06 \mathrm{de}$ & $6.74 \mathrm{~cd}$ & $5.80 \mathrm{C}$ \\
\hline 6000 & $8.00 \mathrm{~h}$ & $11.10 \mathrm{f}$ & $13.35 \mathrm{e}$ & $10.82 \mathrm{D}$ & $3.74 \mathrm{hi}$ & $4.94 \mathrm{fg}$ & $5.81 \mathrm{e}$ & $4.83 \mathrm{D}$ \\
\hline 8000 & $6.62 \mathrm{i}$ & $8.72 \mathrm{~h}$ & $9.98 \mathrm{~g}$ & $8.44 \mathrm{E}$ & $3.10 \mathrm{i}$ & $3.89 \mathrm{~h}$ & $4.34 \mathrm{gh}$ & $3.78 \mathrm{E}$ \\
\hline \multirow[t]{2}{*}{ Mean } & $9.80 \mathrm{C}$ & $12.46 \mathrm{~B}$ & $15.16 \mathrm{~A}$ & & $4.59 \mathrm{C}$ & $5.76 \mathrm{~B}$ & $6.60 \mathrm{~A}$ & \\
\hline & \multicolumn{8}{|c|}{ Second season; 2019} \\
\hline 0.00 & $11.48 \mathrm{e}$ & $16.51 \mathrm{c}$ & $19.75 \mathrm{a}$ & $15.91 \mathrm{~A}$ & $6.14 \mathrm{c}$ & $7.37 b$ & $9.40 \mathrm{a}$ & $7.84 \mathrm{~A}$ \\
\hline 2000 & $8.54 \mathrm{~g}$ & $16.76 \mathrm{c}$ & $18.69 \mathrm{~b}$ & 14.66B & $4.61 \mathrm{~d}-\mathrm{f}$ & $8.18 b$ & $8.92 \mathrm{a}$ & $7.24 \mathrm{~B}$ \\
\hline 4000 & $8.88 \mathrm{~g}$ & $13.97 \mathrm{~d}$ & $16.07 \mathrm{c}$ & $12.98 \mathrm{C}$ & $4.75 \mathrm{de}$ & $6.73 c$ & $7.64 \mathrm{~b}$ & $6.37 \mathrm{C}$ \\
\hline 6000 & $7.54 \mathrm{~h}$ & $10.88 \mathrm{e}$ & $13.41 \mathrm{~d}$ & $10.61 \mathrm{D}$ & $4.05 \mathrm{f}$ & $5.25 \mathrm{~d}$ & $6.38 \mathrm{c}$ & $5.23 \mathrm{D}$ \\
\hline 8000 & $0.00 \mathrm{i}$ & $6.71 \mathrm{~h}$ & $9.54 \mathrm{f}$ & $5.42 \mathrm{E}$ & $0.00 \mathrm{~h}$ & $3.25 \mathrm{~g}$ & $4.52 \mathrm{ef}$ & $2.59 \mathrm{E}$ \\
\hline Mean & $7.29 \mathrm{C}$ & $12.97 \mathrm{~B}$ & $15.49 \mathrm{~A}$ & & $3.91 \mathrm{C}$ & $6.28 \mathrm{~B}$ & $7.37 \mathrm{~A}$ & \\
\hline
\end{tabular}

Mean followed by the same letter in a column or row don't differ significantly according to Duncan's New Multiple Range test

Table 6. Effect of salinity levels, magnetite rates and their interactions on roots fresh and dry weights of Moringa oleifera Lam. transplants during 2018 and 2019 seasons

\begin{tabular}{|c|c|c|c|c|c|c|c|c|}
\hline \multirow{3}{*}{ Salinity level (pprn) } & \multicolumn{4}{|c|}{ Roots fresh weights (g) } & \multicolumn{4}{|c|}{ Roots dry weights (g) } \\
\hline & 0.00 & $2 \mathrm{~g}$ & $4 \mathrm{~g}$ & Mean & 0.00 & $2 \mathrm{~g}$ & $4 \mathrm{~g}$ & Mean \\
\hline & \multicolumn{8}{|c|}{ First season; 2018} \\
\hline 0.00 & $13.38 \mathrm{e}$ & $22.38 b$ & $25.78 \mathrm{a}$ & $20.51 \mathrm{~A}$ & $4.13 \mathrm{f}$ & $7.99 \mathrm{~b}$ & $10.31 \mathrm{a}$ & $7.48 \mathrm{~A}$ \\
\hline 2000 & $13.81 \mathrm{de}$ & $22.42 b$ & $25.73 a$ & $20.65 \mathrm{~A}$ & $4.28 \mathrm{f}$ & $8.02 \mathrm{~b}$ & $10.25 \mathrm{a}$ & $7.52 \mathrm{~A}$ \\
\hline 4000 & $11.23 \mathrm{f}$ & $18.97 \mathrm{bc}$ & $20.39 \mathrm{c}$ & $16.86 \mathrm{~B}$ & $3.46 \mathrm{~g}$ & $6.73 \mathrm{c}$ & $8.13 b$ & $6.10 \mathrm{~B}$ \\
\hline 6000 & $9.23 \mathrm{gh}$ & $13.28 \mathrm{e}$ & $15.33 \mathrm{~d}$ & $12.61 \mathrm{C}$ & $2.85 \mathrm{~h}$ & $4.68 \mathrm{e}$ & $6.12 \mathrm{~d}$ & $4.55 \mathrm{C}$ \\
\hline 8000 & $7.16 \mathrm{j}$ & 8.38hi & $10.62 \mathrm{fg}$ & $8.72 \mathrm{D}$ & $2.20 \mathrm{i}$ & $2.98 \mathrm{~h}$ & $4.19 \mathrm{f}$ & 3.13D \\
\hline \multirow[t]{2}{*}{ Mean } & $10.96 \mathrm{C}$ & $17.09 \mathrm{~B}$ & $19.57 \mathrm{~A}$ & + & $3.38 \mathrm{C}$ & $6.08 \mathrm{~B}$ & $7.80 \mathrm{~A}$ & \\
\hline & \multicolumn{8}{|c|}{ Second season; 2019} \\
\hline 0.00 & $11.63 \mathrm{~b}-\mathrm{d}$ & $12.69 \mathrm{~b}$ & $14.03 \mathrm{a}$ & $12.78 \mathrm{~A}$ & $2.96 \mathrm{~cd}$ & $3.42 \mathrm{~b}$ & $3.89 \mathrm{a}$ & $3.43 \mathrm{~A}$ \\
\hline 2000 & 10.65de & $11.05 \mathrm{c}-\mathrm{e}$ & $12.20 \mathrm{~b}$ & 11.30B & $2.71 \mathrm{de}$ & $2.96 \mathrm{~cd}$ & $3.39 \mathrm{~b}$ & $3.02 \mathrm{~B}$ \\
\hline 4000 & $9.48 \mathrm{fg}$ & $10.23 \mathrm{ef}$ & $11.84 \mathrm{bc}$ & $10.52 \mathrm{~B}$ & $2.37 \mathrm{e}-\mathrm{g}$ & $2.70 \mathrm{de}$ & $3.26 b c$ & $2.78 \mathrm{C}$ \\
\hline 6000 & 7.70hi & $8.43 \mathrm{gh}$ & $9.03 \mathrm{~g}$ & $8.39 \mathrm{C}$ & $1.89 \mathrm{~h}$ & $2.25 f-h$ & $2.52 \mathrm{ef}$ & $2.22 \mathrm{D}$ \\
\hline 8000 & $0.00 \mathrm{j}$ & 7.39hi & $7.30 \mathrm{i}$ & $4.90 \mathrm{D}$ & $0.00 \mathrm{i}$ & $1.95 \mathrm{~h}$ & $2.01 \mathrm{gh}$ & $1.32 \mathrm{E}$ \\
\hline Mean & $7.89 \mathrm{C}$ & $9.96 \mathrm{~B}$ & $10.88 \mathrm{~A}$ & & $1.99 \mathrm{C}$ & $2.66 \mathrm{~B}$ & $3.02 \mathrm{~A}$ & \\
\hline
\end{tabular}

Mean followed by the same letter in a column or row don't differ significantly according to Duncan's New Multiple Range test 
Table 7. Effect of salinity levels, magnetite rates and their interactions on resistance index and benefit coefficient of Moringa oleifera Lam. transplants during 2018 and 2019 seasons

\begin{tabular}{|c|c|c|c|c|c|c|c|c|}
\hline \multirow{3}{*}{ Salinity level (ppm) } & \multicolumn{4}{|c|}{ Salt resistance index $(\%)$} & \multicolumn{4}{|c|}{ Benefit coefficient of $\mathrm{Fe}_{3} \mathrm{O}_{4}(\%)$} \\
\hline & 0.00 & $2 \mathrm{~g}$ & $4 \mathrm{~g}$ & Mean & 0.00 & $2 \mathrm{~g}$ & $4 \mathrm{~g}$ & Mean \\
\hline & \multicolumn{8}{|c|}{ First season; 2018} \\
\hline 0.00 & $100.00 \mathrm{a}$ & $100.00 \mathrm{a}$ & $100.00 \mathrm{a}$ & $100.00 \mathrm{~A}$ & $0.00 \mathrm{~h}$ & $9.57 \mathrm{~g}$ & $38.34 \mathrm{de}$ & $15.97 \mathrm{D}$ \\
\hline 2000 & $93.62 b$ & $94.15 b$ & $89.23 \mathrm{c}$ & $92.33 \mathrm{~B}$ & $0.00 \mathrm{~h}$ & $40.97 \mathrm{~cd}$ & $54.67 \mathrm{~b}$ & $31.88 \mathrm{~A}$ \\
\hline 4000 & $82.03 \mathrm{~d}$ & $81.97 \mathrm{~d}$ & $82.90 \mathrm{~d}$ & $82.30 \mathrm{C}$ & $0.00 \mathrm{~h}$ & $35.20 \mathrm{e}$ & $51.77 \mathrm{~b}$ & 28.99B \\
\hline 6000 & $65.86 f$ & $71.37 \mathrm{e}$ & $68.11 \mathrm{f}$ & $68.45 \mathrm{D}$ & $0.00 \mathrm{~h}$ & $34.74 \mathrm{e}$ & $59.05 \mathrm{a}$ & $31.27 \mathrm{~A}$ \\
\hline 8000 & $45.39 \mathrm{~h}$ & $58.91 \mathrm{~g}$ & $56.33 \mathrm{~h}$ & $53.54 \mathrm{E}$ & $0.00 \mathrm{~h}$ & $27.70 \mathrm{f}$ & $42.57 \mathrm{c}$ & $23.42 \mathrm{C}$ \\
\hline \multirow[t]{2}{*}{ Mean } & $77.38 \mathrm{C}$ & $81.28 \mathrm{~A}$ & 79.61B & & $0.00 \mathrm{C}$ & 29.64B & $49.28 \mathrm{~A}$ & \\
\hline & \multicolumn{8}{|c|}{ Second season; 2019} \\
\hline 0.00 & $100.00 \mathrm{a}$ & $100.00 \mathrm{a}$ & $100.00 \mathrm{a}$ & $100.00 \mathrm{~A}$ & $0.00 \mathrm{j}$ & $27.67 \mathrm{i}$ & $53.87 \mathrm{f}$ & $27.85 \mathrm{E}$ \\
\hline 2000 & $92.09 \mathrm{~b}$ & $88.49 \mathrm{c}$ & $88.10 \mathrm{c}$ & $89.56 \mathrm{~B}$ & $0.00 \mathrm{j}$ & $86.41 \mathrm{~d}$ & $102.5 \mathrm{c}$ & $62.97 \mathrm{~B}$ \\
\hline 4000 & $82.04 \mathrm{~d}$ & $81.89 \mathrm{~d}$ & $85.50 \mathrm{c}$ & $83.14 \mathrm{C}$ & $0.00 \mathrm{j}$ & $40.55 \mathrm{~h}$ & $64.07 \mathrm{e}$ & $34.87 \mathrm{D}$ \\
\hline 6000 & $66.31 \mathrm{e}$ & $68.89 \mathrm{e}$ & $66.22 \mathrm{e}$ & 67.14D & $0.00 \mathrm{j}$ & $48.95 \mathrm{~g}$ & $63.88 \mathrm{e}$ & $37.61 \mathrm{C}$ \\
\hline 8000 & $0.00 \mathrm{~h}$ & $62.06 f$ & $56.33 \mathrm{~g}$ & $39.46 \mathrm{E}$ & $0.00 \mathrm{j}$ & $325.0 \mathrm{~b}$ & $452.0 \mathrm{a}$ & $259.0 \mathrm{~A}$ \\
\hline Mean & $68.09 \mathrm{C}$ & $80.27 \mathrm{~A}$ & $79.23 \mathrm{~B}$ & & $0.00 \mathrm{C}$ & 106.1B & $147.3 \mathrm{~A}$ & \\
\hline
\end{tabular}

Mean followed by the same letter in a column or row don't differ significantly according to Duncan's New Multiple Range test

\section{Chemical composition of the leaves}

It is evident from data presented in Table 8 that the gradual increase of salinity level resulted a gradual decrease in concentrations of chlorophyll $\mathrm{a}, \mathrm{b}$ and carotenoids $(\mathrm{mg} / \mathrm{g}$ f.w.) and the percentages of $\mathrm{N}$ and $\mathrm{P}$ except for $2000 \mathrm{ppm}$ salinity level that raised $\mathrm{P}$ concentration to $0.752 \%$ versus $0.657 \%$ for control. The opposite was the right in relation to $\mathrm{K}, \mathrm{Na}$ and $\mathrm{Cl}$ percentages as well free proline (mg/100 $\mathrm{g}$ d.w.). Which was progressively increased with increasing salinity level.

Magnetite, on the other side, linearly elevated concentrations of the previous constituents as the rate of its application was increased except for $\mathrm{N}$ and $\mathrm{Na}$ percentages which were adversely affected. Also, $\mathrm{Fe}_{3} \mathrm{O}_{4}$ at $4 \mathrm{~g} /$ plant rate diminished $\mathrm{Cl} \%$, whereas $2 \mathrm{~g} /$ plant rate increased it.

The highest concentrations of chlorophyll a, b, carotenoids and nitrogen were attained by interacting between either zero or $2000 \mathrm{ppm}$ salinity and magnetic iron, especially at $4 \mathrm{~g} / \mathrm{plant}$ dose, but the highest percent of phosphorus was obtained from 2000 ppm salinity level + magnetite at either 2 or $4 \mathrm{~g} /$ plant. The opposite was the right concerning $\mathrm{K}, \mathrm{Na}, \mathrm{Cl}$ and proline, as their maximal concentrations were obtained by combining between 8000 ppm salinity and magnetite at $2 \mathrm{~g} / \mathrm{plant}$ in most cases of both seasons. 
Table 8. Effect of salinity levels, magnetite rates and their interactions on some constituent's concentrations in Moringa oleifera Lam. leaves during 2019 seasons

\begin{tabular}{|c|c|c|c|c|c|c|c|c|c|c|c|c|}
\hline \multirow{3}{*}{ Salinity level (ppm) } & \multicolumn{4}{|c|}{ Chlorophyll a (mg/g f.w.) } & \multicolumn{4}{|c|}{ Chlorophyll b (mg/g f.w.) } & \multicolumn{4}{|c|}{ Carotenoids (mg/g f.w.) } \\
\hline & 0.00 & $2 \mathrm{~g}$ & $4 \mathrm{~g}$ & Mean & 0.00 & $2 \mathrm{~g}$ & $4 \mathrm{~g}$ & Mean & 0.00 & $2 \mathrm{~g}$ & $4 \mathrm{~g}$ & Mean \\
\hline & \multicolumn{12}{|c|}{ First season; 2018} \\
\hline 0 & $2.103 \mathrm{~g}$ & $3.384 \mathrm{a}$ & $3.219 \mathrm{c}$ & $2.902 \mathrm{~A}$ & $1.640 \mathrm{f}$ & $1.837 \mathrm{~d}$ & $2.226 \mathrm{a}$ & $1.901 \mathrm{~A}$ & $0.733 a-c$ & $0.810 \mathrm{a}$ & $0.796 \mathrm{ab}$ & $0.780 \mathrm{~A}$ \\
\hline 2000 & $2.04 \mathrm{~g}$ & $3.319 \mathrm{~b}$ & $3.110 \mathrm{~d}$ & $2.823 \mathrm{~B}$ & $1.631 \mathrm{f}$ & $1.755 \mathrm{e}$ & $2.083 b$ & $1.823 \mathrm{~B}$ & $0.734 \mathrm{a}-\mathrm{c}$ & $0.817 \mathrm{a}$ & $0.781 \mathrm{ab}$ & $0.777 \mathrm{~A}$ \\
\hline 4000 & $1.503 \mathrm{~h}$ & $2.233 \mathrm{f}$ & $2.467 \mathrm{e}$ & $2.068 \mathrm{C}$ & $1.36 \mathrm{~g}$ & $1.640 \mathrm{f}$ & $1.937 \mathrm{c}$ & $1.651 \mathrm{C}$ & $0.697 \mathrm{c}$ & $0.810 \mathrm{a}$ & $0.790 \mathrm{ab}$ & $0.766 \mathrm{~A}$ \\
\hline 6000 & $1.299 \mathrm{i}$ & $2.061 \mathrm{~g}$ & $2.201 \mathrm{f}$ & $1.854 \mathrm{D}$ & $1.045 \mathrm{i}$ & $1.321 \mathrm{~h}$ & $1.375 \mathrm{~g}$ & $1.247 \mathrm{D}$ & $0.675 \mathrm{c}$ & $0.724 \mathrm{bc}$ & $0.733-\mathrm{c}$ & $0.711 \mathrm{~B}$ \\
\hline 8000 & $0.00 \mathrm{j}$ & $1.293 \mathrm{i}$ & $1.358 \mathrm{i}$ & $1.326 \mathrm{E}$ & $0.00 \mathrm{j}$ & $1.039 \mathrm{i}$ & $1.050 \mathrm{i}$ & $1.045 \mathrm{E}$ & $0.000 \mathrm{e}$ & $0.501 \mathrm{~d}$ & $0.540 \mathrm{~d}$ & $0.521 \mathrm{C}$ \\
\hline \multirow[t]{3}{*}{ Mean } & $1.736 \mathrm{~B}$ & $2.458 \mathrm{~A}$ & $2.471 \mathrm{~A}$ & & $1.423 \mathrm{C}$ & $1.518 \mathrm{~B}$ & $1.734 \mathrm{~A}$ & & $0.71 \mathrm{~B}$ & $0.733 \mathrm{~A}$ & $0.728 \mathrm{~A}$ & \\
\hline & \multicolumn{4}{|c|}{$\mathbf{N}(\%)$} & \multicolumn{4}{|c|}{$\mathbf{P}(\%)$} & \multicolumn{4}{|c|}{$\mathrm{K}(\%)$} \\
\hline & 0.00 & $2 \mathrm{~g}$ & $4 \mathrm{~g}$ & Mean & 0.00 & $2 \mathrm{~g}$ & $4 \mathrm{~g}$ & Mean & 0.00 & $2 \mathrm{~g}$ & $4 \mathrm{~g}$ & Mean \\
\hline 0 & $4.250 \mathrm{bc}$ & $4.250 \mathrm{bc}$ & $4.310 \mathrm{a}$ & $4.270 \mathrm{~A}$ & $0.651 \mathrm{~cd}$ & $0.658 \mathrm{c}$ & $0.663 c$ & $0.657 \mathrm{~B}$ & 1.0731 & $1.163 \mathrm{k}$ & $1.177 \mathrm{k}$ & $1.138 \mathrm{E}$ \\
\hline 2000 & $4.230 \mathrm{c}$ & $4.240 \mathrm{c}$ & $4.300 \mathrm{ab}$ & $4.260 \mathrm{~A}$ & $0.649 \mathrm{~cd}$ & $0.726 \mathrm{~b}$ & $0.880 \mathrm{a}$ & $0.752 \mathrm{~A}$ & $1.328 \mathrm{j}$ & $1.396 \mathrm{i}$ & $1.465 \mathrm{~h}$ & $1.396 \mathrm{D}$ \\
\hline 4000 & $3.760 \mathrm{f}$ & $3.950 \mathrm{e}$ & $4.070 \mathrm{~d}$ & 3.930B & $0.476 \mathrm{~g}$ & $0.617 \mathrm{e}$ & $0.635 \mathrm{~d}$ & $0.576 \mathrm{C}$ & $1.670 \mathrm{~g}$ & $1.793 \mathrm{f}$ & $1.881 \mathrm{e}$ & $1.781 \mathrm{C}$ \\
\hline 6000 & $3.350 \mathrm{~h}$ & $3.610 \mathrm{~g}$ & $3.780 f$ & $3.580 \mathrm{C}$ & $0.418 \mathrm{hi}$ & $0.479 \mathrm{~g}$ & $0.521 \mathrm{f}$ & $0.473 \mathrm{D}$ & $1.911 \mathrm{~d}$ & $2.013 \mathrm{c}$ & $2.094 \mathrm{~b}$ & $2.006 \mathrm{~B}$ \\
\hline 8000 & $0.000 \mathrm{k}$ & $2.360 \mathrm{j}$ & $2.450 \mathrm{i}$ & $2.410 \mathrm{D}$ & $0.00 \mathrm{j}$ & $0.411 \mathrm{i}$ & $0.429 \mathrm{~h}$ & $0.420 \mathrm{E}$ & $0.00 \mathrm{~m}$ & $2.133 \mathrm{a}$ & $2.148 \mathrm{a}$ & $2.141 \mathrm{~A}$ \\
\hline \multirow[t]{3}{*}{ Mean } & $3.900 \mathrm{C}$ & $3.680 \mathrm{~B}$ & $3.782 \mathrm{~A}$ & & $0.549 \mathrm{C}$ & $0.578 \mathrm{~B}$ & $0.626 \mathrm{~A}$ & & $1.496 \mathrm{C}$ & $1.700 \mathrm{~B}$ & $1.753 \mathrm{~A}$ & \\
\hline & \multicolumn{4}{|c|}{$\mathrm{Na}(\%)$} & \multicolumn{4}{|c|}{$\mathrm{Cl}(\%)$} & \multicolumn{4}{|c|}{ Proline (mg/100 g f.w.) } \\
\hline & 0.00 & $2 \mathrm{~g}$ & $4 \mathrm{~g}$ & Mean & 0.00 & $2 \mathrm{~g}$ & $4 \mathrm{~g}$ & Mean & 0.00 & $2 \mathrm{~g}$ & $4 \mathrm{~g}$ & Mean \\
\hline 0 & $0.859 \mathrm{~g}$ & $0.730 \mathrm{j}$ & $0.730 \mathrm{j}$ & $0.773 \mathrm{E}$ & $1.170 \mathrm{~g}$ & $1.170 \mathrm{~g}$ & $1.100 \mathrm{~h}$ & $1.147 \mathrm{E}$ & 5.9761 & 5.9101 & $5.646 \mathrm{~m}$ & $5.844 \mathrm{E}$ \\
\hline 2000 & $1.013 \mathrm{f}$ & $0.783 \mathrm{i}$ & $0.771 \mathrm{i}$ & $0.856 \mathrm{D}$ & $0.760 \mathrm{e}$ & $1.460 \mathrm{f}$ & $1.430 f$ & $1.550 \mathrm{D}$ & $9.735 \mathrm{i}$ & $9.315 \mathrm{j}$ & $9.000 \mathrm{k}$ & $9.350 \mathrm{D}$ \\
\hline 4000 & $1.079 \mathrm{e}$ & $0.811 \mathrm{~h}$ & $0.780 \mathrm{i}$ & $0.890 \mathrm{C}$ & $2.050 \mathrm{~d}$ & $2.050 \mathrm{~d}$ & $1.790 \mathrm{e}$ & $1.963 \mathrm{C}$ & $20.284 f$ & $17.391 \mathrm{~g}$ & $17.110 \mathrm{~h}$ & $18.262 \mathrm{C}$ \\
\hline 6000 & $1.303 c$ & $1.110 \mathrm{~d}$ & $1.101 \mathrm{~d}$ & 1.171B & $2.630 \mathrm{a}$ & $2.350 \mathrm{c}$ & $2.310 \mathrm{c}$ & $2.430 \mathrm{~B}$ & $41.500 \mathrm{~b}$ & $35.581 \mathrm{~d}$ & $31.532 \mathrm{e}$ & 36.204B \\
\hline 8000 & $0.000 \mathrm{k}$ & $1.567 \mathrm{a}$ & $1.433 b$ & $1.500 \mathrm{~A}$ & $0.00 \mathrm{i}$ & $2.610 \mathrm{a}$ & $2.500 \mathrm{~b}$ & $2.555 \mathrm{~A}$ & $0.00 \mathrm{n}$ & $43.610 \mathrm{a}$ & $38.336 \mathrm{c}$ & $40.973 \mathrm{~A}$ \\
\hline Mean & $1.064 \mathrm{~A}$ & $1.000 \mathrm{~B}$ & $0.963 \mathrm{C}$ & - & $1.903 \mathrm{AB}$ & $1.928 \mathrm{~A}$ & $1.826 \mathrm{~B}$ & & $19.374 \mathrm{C}$ & $22.361 \mathrm{~A}$ & $20.325 B$ & \\
\hline
\end{tabular}

Mean followed by the same letter in a column or row don't differ significantly according to Duncan's New Multiple Range test 


\section{Discussion}

\section{Effect of soil salinity, magnetite and their interaction on vegetative and root growth parameters}

The adverse effects of saline soil on growth may be attributed to either low water uptake due to low potential of soil water (Munns, 2002) or certain ion toxicity $\left(\mathrm{Na}^{+}\right.$and $\mathrm{Cl}^{-}$) or both (Carter et al., 2005). This fact was emphasized by Elhag and Abdalla (2014) who stated that salinity depresses plant growth via affecting water absorption and biochemical processes such as $\mathrm{N}, \mathrm{CO}_{2}$ assimilation and protein biosynthesis or accumulated high concentration of potentially toxic ions $\left(\mathrm{Na}^{+}\right.$and $\left.\mathrm{Cl}^{-}\right)$. Jose et al. (2016) ascribed the reduction in growth by salinity to the effect of osmotic stress and the inhibition of cell division rather than cell expansion coupled with the great reduction in photosynthesis. High salinity also leads to leaf abscission due to ion accumulation in the leaves, particularly the oldest ones. Reduction of growth by salinity may be attributed to a decrease in all volume at a constant cell number. Mechanism of salt may result in inhibition of cell division, consequently reduces the rate of plant development (Khan et al., 2009). Jou et al. (2006), however suggested that ATPase participates in the endoplasmic Reticulum-Golgi mediated, protein sorting machinery for both housekeeping function and compartmentalization of excess $\mathrm{Na}^{+}$under high salinity. Such truth was aforementioned by Munns (2002) who proposed that salt tolerant plants may have a low rate of $\mathrm{Na}^{+}$and $\mathrm{Cl}^{-}$transport to leaves beside their ability to compartmentalize these ions in vacuoles to prevent their buildup in the cytoplasm or cell walls and thus avoid salt toxicity. The previous results are in accordance with those postulated by Elhag and Abdalla (2014) who found that stem length and No. leaves of 2 -months-old moringa transplants were significantly reduced (30 and $40 \%$ successively) by the highest $\mathrm{NaCl}$ concentration $(0.8 \%)$. Hence, the 2-months-old moringa transplants can tolerate $\mathrm{NaCl}$ concentration up to $0.4 \%$. This tolerance may be attributed to its ability to restrict the transport of toxic $\mathrm{Na}^{+}$and $\mathrm{Cl}^{-}$ions to the shoot at such lower concentration and their accumulation in the older leaves at higher ones to get rid of them by shedding of those older leaves. So, the mechanism of salinity tolerance of moringa may be the avoidance of toxic ions. Likewise, Cassaniti et al. (2013) claimed that brackish water can be commercially used for production of Chrysanthemum morifolium, Dianthus caryophyllus, Gerbera jamesonii, Hippeastrum vittatum and Anthurium andreanum, up to $2500 \mathrm{ppm}$. Similarly, were those results of Shahin et al. (2008) on Ficus macrocarpa var. Hawaii and Euonymus Japonica cv. Mediopicta, Shahin et al. (2013) on Ficus benjamina cv. Samanthaand Schefflera arboricola cv. Gold Capella, El-Fouly et al. (2015) on Iris tingitana cv. Wedgewood, Jose et al. (2016) on Eucalyptus urophylla and the hybrid of E. urophylla x E. grandis Shahin et al. (2017) affirmed that a gradual decrement in vegetative and root growth of both Casuarina equisetifolia and Eucalyptus rostrata seedlings was observed due to the gradual increment in salinity concentration from 5000 to $15000 \mathrm{ppm}$.

On the other hand, the positive effect of magnetic iron on growth of plants suffered from salt stress may be due to its role in promoting the uptake of $\mathrm{N}, \mathrm{P}, \mathrm{K}$ and $\mathrm{Fe}$ which stimulates plant growth against the toxicity of $\mathrm{Na}^{+}$and $\mathrm{Cl}^{-}$ions that inhibit it. It induces cell metabolism and mitosis of meristematic cells (Barage et al., 2009). It is believed that new protein bands are formed in plants that are treated with $\mathrm{Fe}_{3} \mathrm{O}_{4}$ and these proteins are responsible for the increased growth (Hozyan and Abdul-Qodos, 2010). Furthermore, it declines the hydration of salt ions and colloids, increasing salt solubility, 
and finally leading to leaching such salts from the soil. In the iron atom, there is a number of valence electrons that generates a magnetic field influence on the biochemical processes in plants and renders the roots to exhibit symptoms of magnetism that kills nematodes and injurious bacteria (Yuliando et al., 2016). The aforementioned gains are in harmony with those obtained by Dos Santos et al. (2011) and Nouman et al. (2012b) on Moringa oleifera and Moustafa et al. (2017) who pointed out that seedlings length, No. leaves, root length, No. roots and aerial parts and roots fresh and dry weights of Moringa oleifera seedling subjected to salinity up to $8000 \mathrm{ppm}$ were improved in response to applying $\mathrm{Fe}_{3} \mathrm{O}_{4}$ at $2 \mathrm{~g} /$ pot. Supporting results for this study were also obtained by Abdel-Fattah (2014) on Jacaranda acutifolia, Ahmed et al. (2016) on Acalypha wilkesiana, Shahin et al. (2018) on Terminalia arjuna and El-Sayed et al. (2019) on elephant's ear tree.

\section{Salt resistance index (SRI \%) and benefit coefficient of magnetite (B. Coe. \%).}

These results are reasonable because magnetite usually solubilizes and leaches salts from the soil, and this gives roots a chance to penetrate and distribute well through the growing medium, consequently uptake enough water and nutrients necessary for good and healthy growth. On the same line were those results attained by Al-Qubaie et al. (2003) on Ficus benghalensis, Bougainvillea glabra, Jasminum azoricum, Conocarpus erectus, Tamarix articulata and Ziziphus spina-christi, Shahin et al. (2008) on Ficus macrocarpa Var. Hawaii and Euonymus Japonica cv. Mediopicta, Abdel-Fattah et al. (2012a) on Ficus retusa, Abdel-Fattah et al. (2012b) on Ficus benjamina and El-Sayed et al. (2019) on Enterolobium contortisiliquum.

\section{Chemical composition of the leaves}

The previous data showed the effect of the gradual increase of salinity level may be reasonable because salts usually reduces the water potential of soil solution, consequently decreases minerals and water uptake by roots, accompanied by a depression of photosynthesis and enzymes activity (Munns, 2002). It was also remarkable that accumulation of some amino acids and amides in the leaves and roots of salinity-stressed plants may be attributed to de novo synthesis by protein sorting machinery and not the result of protein degradation (Jou et al., 2006).

On the other hand, improving constituent's concentrations by magnetic iron may indicate its role in repairing salinity harms by reducing $\mathrm{Na}^{+}$and $\mathrm{Cl}^{-}$toxic ions in the medium, coupled with increasing the free proline formation which improves as a colloid water balance in plant cells (Mostafazadeh et al., 2012). Further, $\mathrm{Fe}_{3} \mathrm{O}_{4}$ may create a high energy magnetic field in the root medium which may increase the solubility of $\mathrm{N}$, $\mathrm{P}, \mathrm{K}$ and $\mathrm{Fe}$ nutrients and this in turn promotes the absorption of such minerals, improving ultimately plant growth (Yuliando et al., 2016).

These results, showed a similar trend to those of Elhag and Abdalla (2014) and Moustafa et al. (2017) on Moringa oleifera, Ahmed et al. (2016) on Acalypha wilkesiana, Shahin et al. (2018) on Terminalia arjuna and El-Sayed et al. (2019) who observed that chlorophyll a, b, carotenoids and total carbohydrates concentrations were increased in the leaves of Enterolobium contortisiliquum under salt stress by application of $\mathrm{Fe}_{3} \mathrm{O}_{4}$ at either 2 or $4 \mathrm{~g} /$ plant. 


\section{Conclusion}

From the previous findings, it can be proposed to apply magnetic iron at the rate $4 \mathrm{~g} / \mathrm{plant}$ for enhancing growth and quality of Moringa oleifera seedlings under salt stress up to $6000 \mathrm{ppm}$.

\section{REFERENCES}

[1] Abdel-Fattah, G., Shahin, S. M., Khenizy, S. A. M. (2012a): Improving tolerance of Ficus benjamina L. plant to saline water stress by VAM (Glomus sp.). - J. Biol. Chem. \& Environ. Sci. 7(1): 1-16.

[2] Abdel-Fattah, G., Shahin, S. M., Abdel-Moniem, A. M. (2012b): Could phosphorene help Ficus retosa L. plant to tolerate the deleterious effects of saline water? - J. Biol. Chem. \& Environ. Sci. 7(1): 17-32.

[3] Abdel-Fattah, G. (2014): The role of magnetic iron and sodium selenate in minimizing soil salt hazards on growth and quality of Jacaranda acutifolia Tumb. \& Bionol seedlings. - Sci. J. Flowers \& Ornam. Plants 1(3): 187-198.

[4] Ahmed, M., Abdel-Fattah, G., Shahin, S. (2016): The role of magnetic iron in enhancing the ability of Acalypha wilkesiana Müll. Arg. transplants to tolerate soil salinity. - J. Plant Production, Mansoura Univ. 7(3): 379-384.

[5] Aiyelaagbe, I. O. (2011): Nigerian Horticulture: Facing the Challenges of Human Health and Agricultural productivity. - Keynote address Presented at the $29^{\text {th }}$ Annual National Conf. of Hort. Soc. of Nigeria (HORTSON), 24-29 July; pp. 131-148.

[6] Al-Qubaie, A. I., Shahin, S. M., Abdel-Samad, F. A. (2003): Effect of diluted sea water on growth and chemical composition of some ornamental trees and shrubs. - Egypt J. Appl. Sci. 18(8B): 587-601.

[7] Barage, L. F., da Sousa, M. P., Almeida, T. A. (2009): Enterolobium schomburgkii Benth. Seed germination under saline stress and polyamine application. - Revista Braz Plantas Med. 11(1): 1516-1527.

[8] Batels, L. S., Walden, R. P., Teare, T. D. (1973): Rapid determination of free proline under water stress studies. - Plant and Soil 939: 205-207.

[9] Blake, C. A. (1965): Methods of Soil Analysis Part 1 Physical and Mineralogical Properties Including a Statistics of Measurement and Sampling. - Amr. Soc. Agron. Inc. Pub., Wisconsin., U.S.A.

[10] Carter, C. T., Grieve, C. M., Poss, J. A. (2005): Salinity effects on emergence, survival and ion accumulation of Limonium perezii. - J. Plant Nutrition 28: 1243-1257.

[11] Cassniti, C., Romano, D., Hop, M. E., Flowers, T. J. (2013): Growing floricultural crops with brackish water. - Environmental and Experimental Botany 92: 165-175.

[12] Dos Santos, A. R. F., Mann, R., Ferreira, R. A., de Souza Brito, A. (2011): Water prehydration as priming for Moringa oleifera Lam. seeds under salt stress. - Tropical and Subtropical Agroecosystems 14: 201-207.

[13] El-Fouly, A. S., Abdel-Sattar, M. M., Shahin, S. M. (2015): Effect of different salinity levels, PP-333 treatments and their interaction on growth, flowering, bulbs productivity and chemical composition of Iris tingitana cv. Wedgewood. - J. Agric. Res., Kafr ElSheikh Univ. 41(1): 347-369.

[14] El-Sayed, M. A., El-Fouly, A. S., Shahin, S. M. (2019): Reducing the harmful effect of saline irrigation water on growth and quality of Enterolobium contortisiliquum (elephant`s ear) seedlings. - Bull Fac. Agric., Cairo. Univ. 70: 267-276.

[15] Elhag, A. Z., Abdalla, M. H. (2014): Investigation of sodium chloride tolerance of Moringa oleifera Lam. transplants. - Universal J. Agric. Res. 2(2): 45-49.

[16] Fahey, J. W. (2005): Moringa oleifera: A review of the medical evidence for its nutritional, therapeutic and prophylactic properties. - Trees for Life J. 1(1): 5. 
[17] Hozyan, M., Abdul-Qodos, A. (2010): Irrigation with magnetized water enhances growth, chemical constituent and yield of chickpea (Cicer arietinum L.). - Agric. and Biol. J. of Amer. 1(4): 671-676.

[18] Jackson, M. H. (1973): Soil Chemical Analysis. - Prentice-Hall of India Private Limited M-97, New Delhi, India, 498p.

[19] Jose, A. C., Silva, N. C. N., Faria, J. M., Pereira, W. V. (2016): Influence of osmotic conditioning of Eucalyptus spp. seeds in the tolerance to salt stress. - J. Seed Sci. 38(4): 329-334.

[20] Jou, Y., Chiang, C., Jauh, G., Yen, H. (2006): Functional characterization of ice plant, on AAA-type ATPase associated with the endoplasmic reticulum-Golgi network, and its role in adaptation to salt stress. - Plant Physiol. 141(1): 135-146.

[21] Khan, G. S., Khan, Z. H., Imran, J., Quraishi, M. U., Yaqoob, S., Khan, S. H. (2009): Effect of salinity on germination and growth of some forest tree species at seedling growth. - J. Agric. Res. 47(3): 271-279.

[22] Luatanab, F. S., Olsen, S. R. (1965): Test of an ascorbic acid method for determining phosphorus in water and $\mathrm{NaHCO}_{3}$ extracts from soil. - Soil Sci. Soc. Amer. Proc. 29: 677-678.

[23] McConnachie, G. L., Folkard, G. K., Mtawali, M. A., Sutherland, J. P. (1999): Field trails of appropriate hydraulic flocculation processes. - Water Res. 33(6): 1425-1434.

[24] Mead, R., Curnow, R. N., Harted, A. M. (1993): Statistical Methods in Agriculture and Experimental Biology. $-2^{\text {nd }}$ Ed., Chapman \& Hall Ltd., London, 335p.

[25] Mostafazadeh, B., Khoshrovesh, M., Mousove, S. F., Kiani, A. R. (2012): Effects of magnetized water on soil chemical components underneath trick irrigation. - Amer. Soc. of Civil Engineers: http://dx.doi.org/10.1061/(ASCE) IR. 1943-4774.0000513.

[26] Moustafa, H. E. B., Abdel-Fattah, G., Shahin, S. M. (2017): Germination of Moringa oleifera Lam seeds under salinity conditions in the presence of magnetic iron. - J. Biol. Chem. \& Environ. Sci. 12(3): 551-566.

[27] Munns, R. (2002): Comparative physiology of salt and water stress. - Plant Cell and Environment 25: 239-250.

[28] Nouman, W., Siddiqui, M. T., Basra, S. M., Afzal, I., Rehman, H. (2012a): Enhancement of emergence potential and stand establishments of Moringa oleifera Lam. by seed priming. - Turk J. Agric. For. 36: 227-235.

[29] Nouman, W., Siddiqui, M. T., Basra, S. M., Afzal, I., Rehman, H. (2012b): Response of Moringa oleifera to saline conditions. - Inter. J. Agric. \& Biol. 14(5): 757-762.

[30] Rivas, R., Oliveira, M. T., Santos, M. G. (2013): Three cycles of water deficit from seed to young plants of Moringa oleifera woody species improves stress tolerance. - Plant Physiol. \& Biochem. 63(1): 200-208.

[31] SAS Institute. (2009): SAS/STAT User`s Guides Statistics. - Vers. 6.04, $4^{\text {th }}$ Ed., SAS Institute Inc. Cary, N.C., USA.

[32] Shahin, S. M., Naglaa, E. Y., Mahmoud, A. M. A., El-Feky, A. H. (2008): How far diluted sea water can be used for irrigation of some pot plants. - Proc. $9^{\text {th }}$ Inter. Conf. on Dry Land Development, 7-10 Nov., Organized by IDDC, Alex., Egypt, pp. 363-372.

[33] Shahin, S. M., El-Fouly, A. S., Abdel-Sattar, M. M. (2013): Response of F. benjamina L. cv. Samantha and Schefflera arboricola Endl cv. Gold Copella plants cultivated in two different growing media to salinity of irrigation water. - J. Biol. Chem. \& Environ. Sci. 8(2): 201-218.

[34] Shahin, S. M., Ahmed, M., Tawila, A. S. (2017): Effect of saline irrigation water on growth and quality of Casuarina equisetifolia L. and Eucalyptus rostrata Schlecht plants grown in some soils of Egypt. - Middle East J. Appl. Sci. 7(3): 460-471.

[35] Shahin, S. M., Aly, A. M., Helaly, A. A. E. (2018): Germination of Indian almond (Terminal arjuna Roxb.) seeds as affected by soil salinity in presence or absence of magnetic iron. - J. Plant Production, Mansoura Univ. 9(4): 417-422. 
[36] Steel, R. G. D., Torrie, J. H. (1980): Principles and Procedures of Statistics. - McGrow Hill Book Co., Inc., New York, pp. 377-400.

[37] Suein, W. A. (2008): Moringa oleifera climate, using Moringa oleifera as a source for production of bio-diesel from perennials. - A report by Agric. and Food Dept., Government of Western Australia, 5p.

[38] Sumanta, N., Haque, C. I., Nishika, J., Suprakash, R. (2014): Spectrophotometric analysis of chlorophyllous and carotenoids from commonly grown fern sp. by using various extracting solvents. - Research J. Chem. Sci. 4(9): 63-69.

[39] Thurber, M. D., Fahey, J. W. (2009): Adoption of Moringa oleifera to combat under nutrition viewed through the lens of the "Diffusion of Innovations" theory. - Ecol. Food. Nutr. 48: 212-225.

[40] Wu, L., Huff, D. R. (1983): Characteristics of creeping bentgrass clones (Agrostis stolonifera L.) from a salinity tolerant population after surviving drought stress. HostScience 18(6): 883-885.

[41] Yuliando, A., Astuti, B., Amalia, S. R. (2016): Enhancement of iron content in spinach plants stimulated by magnetic nano particles. - Proc. $3^{\text {rd }}$ Inter Conf. on Advanced Materials Science and Technology (ICAMST), 19 April; 205p., available at: http//doi.org./10.1063/1.4945554. 